\title{
Tratamento endovascular com endoprótese aórtica para aneurisma de artéria subclávia secundário à fístula arteriovenosa axilo-axilar traumática tardia
}

\author{
Endovascular treatment with aortic endoprostheses for subclavian artery aneurysm \\ secondary to late traumatic axillary-axillary arteriovenous fistula
}

Vinicius Tadeu Ramos da Silva Grillo ${ }^{1}$ (D), Rodrigo Gibin Jaldin, Felipe Damascena Rosa ${ }^{1}$ (D),

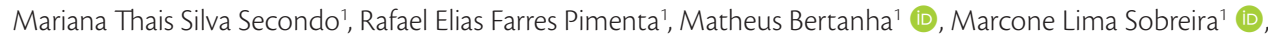

Winston Bonetti Yoshida ${ }^{\top}$

\section{Resumo}

As fístulas arteriovenosas (FAVs) traumáticas envolvendo os vasos axilares e subclávios são incomuns e correspondem de 5 a 10\% de todos os traumas arteriais. A anatomia complexa dessa região torna desafiador o tratamento desse segmento. Neste desafio terapêutico, descrevemos o caso de um homem de 73 anos, encaminhado por edema progressivo e úlcera no membro superior direito, com história pregressa de ferimento por arma de fogo na região infraclavicular direita há cerca de 50 anos. Foi realizada angiotomografia e identificou-se FAV axilo-axilar associada à tortuosidade e dilatação aneurismática de artéria subclávia a jusante. O paciente foi submetido à intervenção endovascular com endoprótese cônica (monoilíaca) $26 \times 14 \times 90$ mm Braile na artéria subclávia aneurismática, posterior à saída da artéria vertebral direita, e endoprótese monoilíaca $16 \times 16 \times 95$ mm Excluder ${ }^{\circledR}$ com sobreposição na primeira prótese, apresentando resultado satisfatório. Portanto, descreve-se a possibilidade de utilização de endoprótese aórtica em situação incomum e de exceção, com sucesso.
\end{abstract}

Palavras-chave: fístula arteriovenosa; artéria subclávia; lesões do sistema vascular; procedimentos endovasculares.

\begin{abstract}
Traumatic arteriovenous fistulas (AVFs) involving the axillary and subclavian vessels are uncommon and account for 5 to $10 \%$ of all arterial traumas. The complex anatomy of this region makes treatment of this segment challenging. In this therapeutic challenge, we describe the case of a 73-year-old man, referred for progressive edema and ulceration involving the right upper limb and with a history of gunshot wound to the right infraclavicular region about 50 years previously. Angiotomography was performed and an axillary-axillary AVF was found, associated with tortuosity and aneurysmatic dilation of the subclavian artery downstream. He underwent endovascular intervention and a conical (monoiliac) $26 \times 14 \times 90 \mathrm{~mm}$ Braile ${ }^{\oplus}$ endoprosthesis was used in the aneurysmatic subclavian artery, posterior to the exit of the right vertebral artery and a $16 \times 16 \times 95 \mathrm{~mm}$ Excluder monoiliac endoprosthesis was placed overlapping the first prosthesis, showing a satisfactory result. Therefore, the possibility of successfully using aortic endoprostheses in an unusual and exceptional situation is described.
\end{abstract}

Keywords: arteriovenous fistula; subclavian artery; vascular system injuries; endovascular procedures.

Como citar: Grillo VTRS, Jaldin RG, Rosa FD, et al. Tratamento endovascular com endoprótese aórtica para aneurisma de artéria subclávia secundário à fístula arteriovenosa axilo-axilar traumática tardia. J Vasc Bras. 2021;20:e20210016. https://doi.org/10.1590/1677-5449.210016

\footnotetext{
${ }^{1}$ Universidade Estadual "Júlio de Mesquita Filho" - UNESP, Faculdade de Medicina de Botucatu, Hospital das Clínicas, Botucatu, SP, Brasil. Fonte de financiamento: Nenhuma.

Conflito de interesse: Os autores declararam não haver conflitos de interesse que precisam ser informados.

Submetido em: Maio 10, 2021. Aceito em: Outubro 28, 2021.
}

O estudo foi realizado no Hospital das Clínicas, Faculdade de Medicina de Botucatu, Universidade Estadual “Júlio de Mesquita Filho” (UNESP), Botucatu, SP, Brasil. 


\section{INTRODUÇÃO}

As fístulas arteriovenosas (FAVs) traumáticas envolvendo os vasos axilares são incomuns ${ }^{1}$, assim como no território subclávio ${ }^{2,3}$, e as FAVs nessas regiões correspondem de 5 a $10 \%$ dos traumas arteriais em civis ${ }^{4}$. A principal etiologia é o trauma penetrante $^{3}$, sendo o ferimento por arma de fogo associado em $65,2 \%$ dos casos, e as lesões mais comumente encontradas foram pseudoaneurisma e $\mathrm{FAV}^{5,6}$. Apesar de incomuns, associam-se a elevada morbimortalidade ${ }^{3,7}$, com altas taxas de óbito ainda no ambiente pré-hospitalar, principalmente devido a choque hemorrágico ${ }^{1}$.

Quando essas lesões vasculares não são diagnosticadas no momento do trauma, os pacientes podem evoluir assintomáticos por anos até que as manifestações clínicas das FAVs sejam identificadas: sopros e frêmitos contínuos com reforço sistólico na topografia dos vasos, circulação colateral venosa, insuficiência cardíaca, diminuição da perfusão do membro ipsilateral e sinais de hipertensão venosa como edema, podendo chegar a ulcerações e gangrena em casos avançados ${ }^{1,2,7-9}$.

A anatomia complexa dessa região e a gravidade clínica do paciente são fatores que aumentam as taxas de morbimortalidade ${ }^{1,3}$, tornando desafiador o tratamento desse segmento para o cirurgião ${ }^{10}$. Este estudo foi devidamente avaliado e aprovado pelo Comitê de Ética em Pesquisa (CAAE 45890921.0.0000.5411, parecer número 4.699.405).

\section{Parte I - situação clínica}

Um homem de 73 anos foi encaminhado por história de edema progressivo no membro superior direito (MSD) associado à úlcera de estase venosa na mão direita há 6 meses. Apresentava antecedente de hipertensão arterial, em uso de losartana, sem outras comorbidades, e relatou história pregressa de ferimento por arma de fogo na região infraclavicular direita há cerca de 50 anos, à época sem maiores repercussões, intervenções cirúrgicas ou diagnósticas. O paciente notou aparecimento progressivo de veias varicosas na topografia do orifício de entrada do projétil na região infraclavicular direita, iniciado após o trauma e que aumentou em número e calibre no decorrer dos anos. Ao exame físico, apresentava-se com edema de ombro, antebraço e mão direita, associado a veias varicosas desde antebraço até região infraclavicular, escurecimento da mão, áreas de necrose seca no $1^{\circ}$ e $3^{\circ}$ quirodáctilos e grande ulceração em face dorsal da mão (Figura 1). O membro superior esquerdo e os membros inferiores bilateralmente não apresentavam edema ou lesões tróficas. Os pulsos radiais, ulnares e distais de membros inferiores eram palpáveis e amplos.

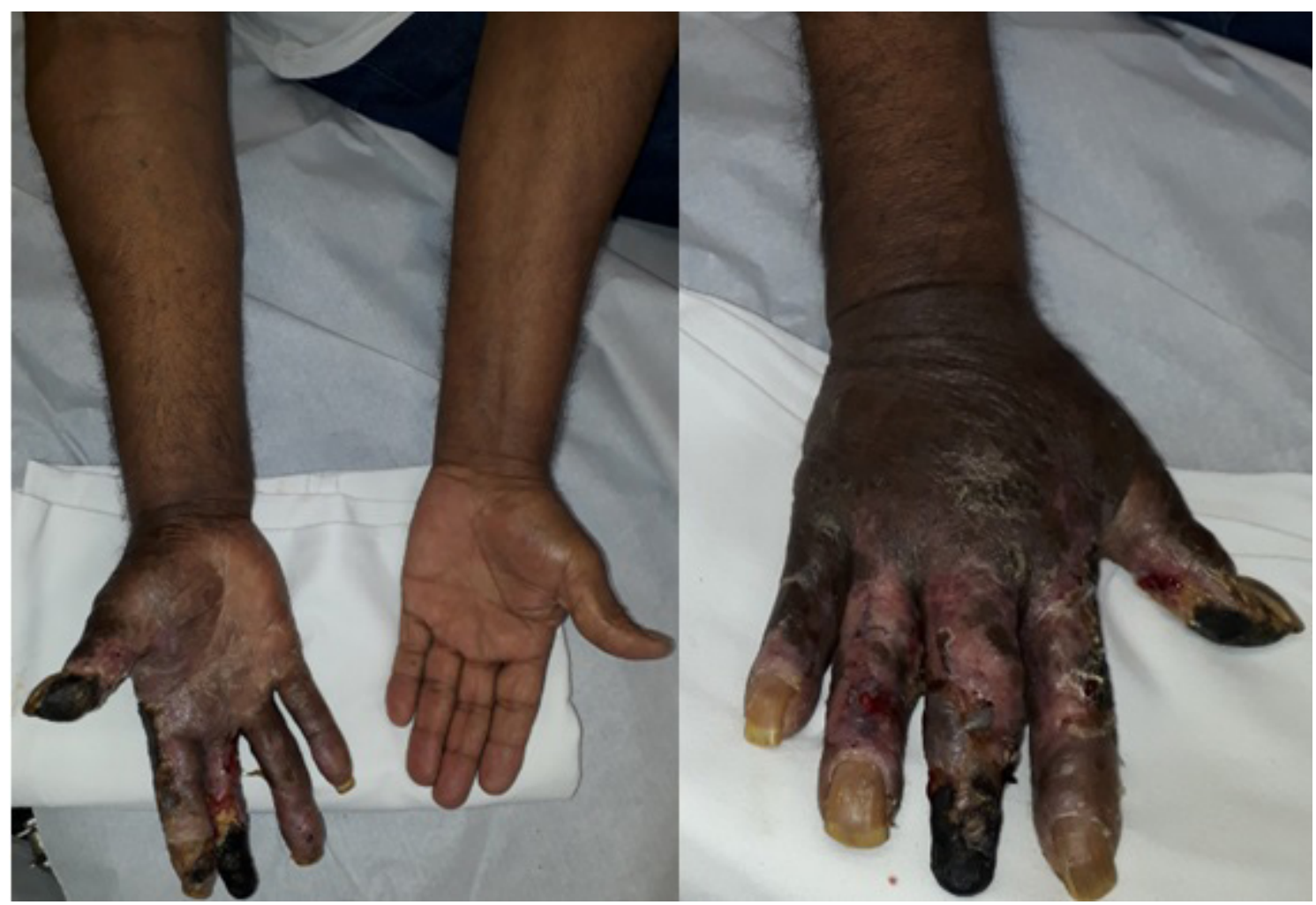

Figura 1. Aspecto inicial das lesões no momento da admissão. Nota-se edema de membro superior direito associado a escurecimento da mão e antebraço e lesões tróficas. 
$\mathrm{Na}$ topografia infraclavicular direita, auscultavase sopro contínuo com reforço sistólico. Quanto aos exames laboratoriais de entrada, hemograma, plaquetas, função renal, creatinofosfoquinase (CPK), coagulograma e eletrólitos estavam normais. O paciente foi submetido à angiotomografia de tórax, abdômen e MSD (Figura 2), sendo identificada FAV axilo-axilar associada à tortuosidade e dilatação aneurismática de artéria subclávia a jusante, com maior diâmetro de $24 \mathrm{~mm}$ e extensão $125 \mathrm{~mm}$.

Caso indicada a abordagem cirúrgica, as opções de tratamento seriam:

- Cirurgia aberta convencional, com toracotomia e ligadura da FAV ou aneurismectomia com derivação arterial;

- Abordagem endovascular e embolização da FAV com plugue vascular;

- Abordagem endovascular e utilização off-label de endoprótese aórtica devido ao diâmetro e à extensão da artéria subclávia.

\section{Parte II - o que foi feito}

Foi decidido pela intervenção endovascular para evitar toracotomia. Realizou-se incisão longitudinal na face medial do terço proximal do braço direito

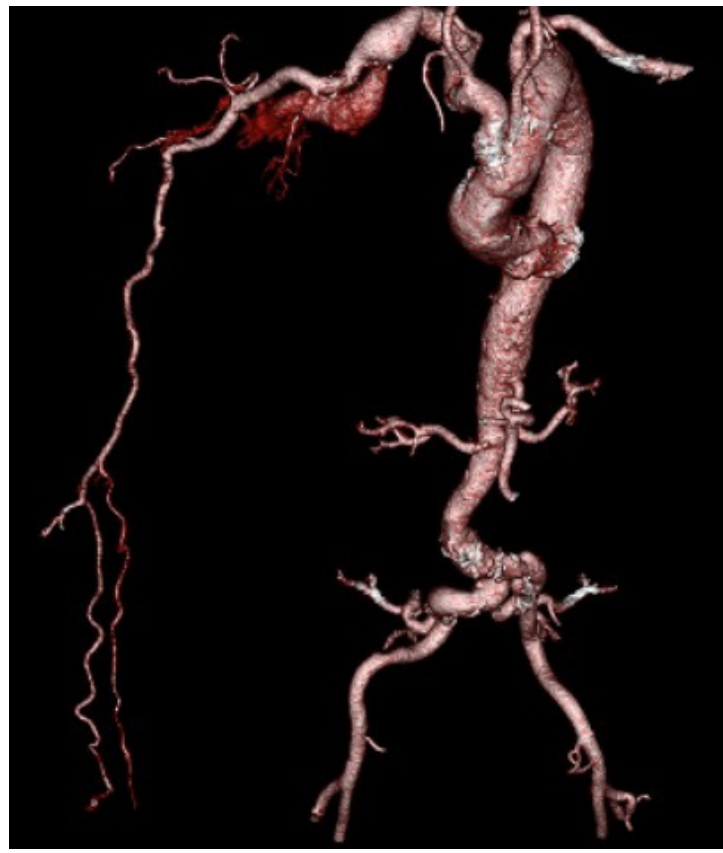

Figura 2. Reconstrução tridimensional da angiotomografia, evidenciando FAV axilo-axilar direita associada à tortuosidade e aneurisma de artéria subclávia direita a jusante. e reparo da artéria braquial (ABD). Foi realizada punção da transição axilo-braquial sob visão direta (diâmetro da artéria axilar de aproximadamente $6 \mathrm{~mm}$ ) e passagem do introdutor $6 \mathrm{~F}$, transposição da lesão com fio guia 0,035" Stiff Roadrunner ${ }^{\circledR}$ (Cook Medical, Bloomington, Ind) sob cateter vertebral 5F, posicionando-o na aorta ascendente. Trocou-se o fio guia pelo Super-stiff Lunderquist ${ }^{\circledR}$ (Cook Medical, Bloomington, Ind), e foi retirado o cateter vertebral. Por punção retrógrada de artéria femoral comum direita e passagem do introdutor $5 \mathrm{~F}$, foi cateterizado o tronco braquiocefálico com fio guia 0,035 " Stiff Roadrunner $^{\circledR}$ (Cook Medical, Bloomington, Ind) sob cateter Simmons 2 (Cook Medical, Bloomington, Ind) (Figura 3). Na via MSD, foi feito implante de endoprótese cônica (monoilíaca) $26 \times 14 \times 90 \mathrm{~mm}$ de perfil 18Fr Braile ${ }^{\circledR}$ (Braile Biomédica, São José do Rio Preto, Brasil) na artéria subclávia aneurismática, posterior à saída da artéria vertebral direita (AVD) (Figura 4). Na sequência, foi liberada uma extensão de ramo de endoprótese bifurcada de $16 \times 16 \times 95 \mathrm{~mm}$ Excluder $^{\circledR}$ (WL Gore \& Associates, Flagstaff, EUA) (Figura 5) com sobreposição na primeira prótese. Evidenciou-se endoleak tipo 3 na imagem de controle, sendo totalmente tratado por balonamento com balão

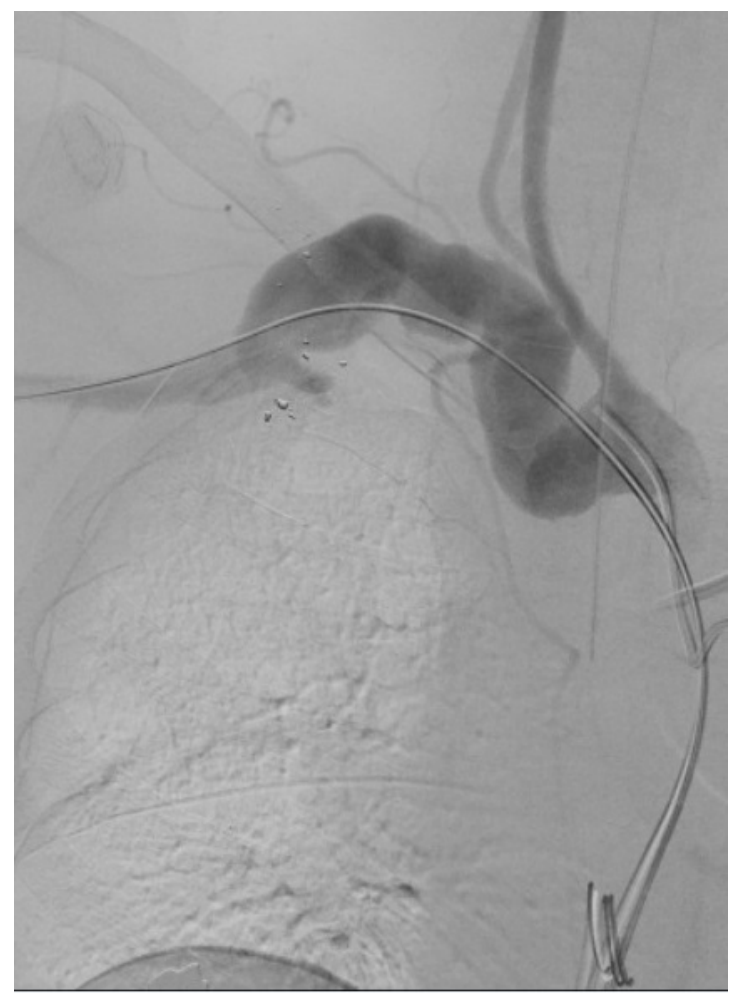

Figura 3. Angiografia por subtração digital evidenciando artéria subclávia direita tortuosa e com dilatação aneurismática. Nota-se estilhaços do projétil de arma de fogo na topografia da lesão. 


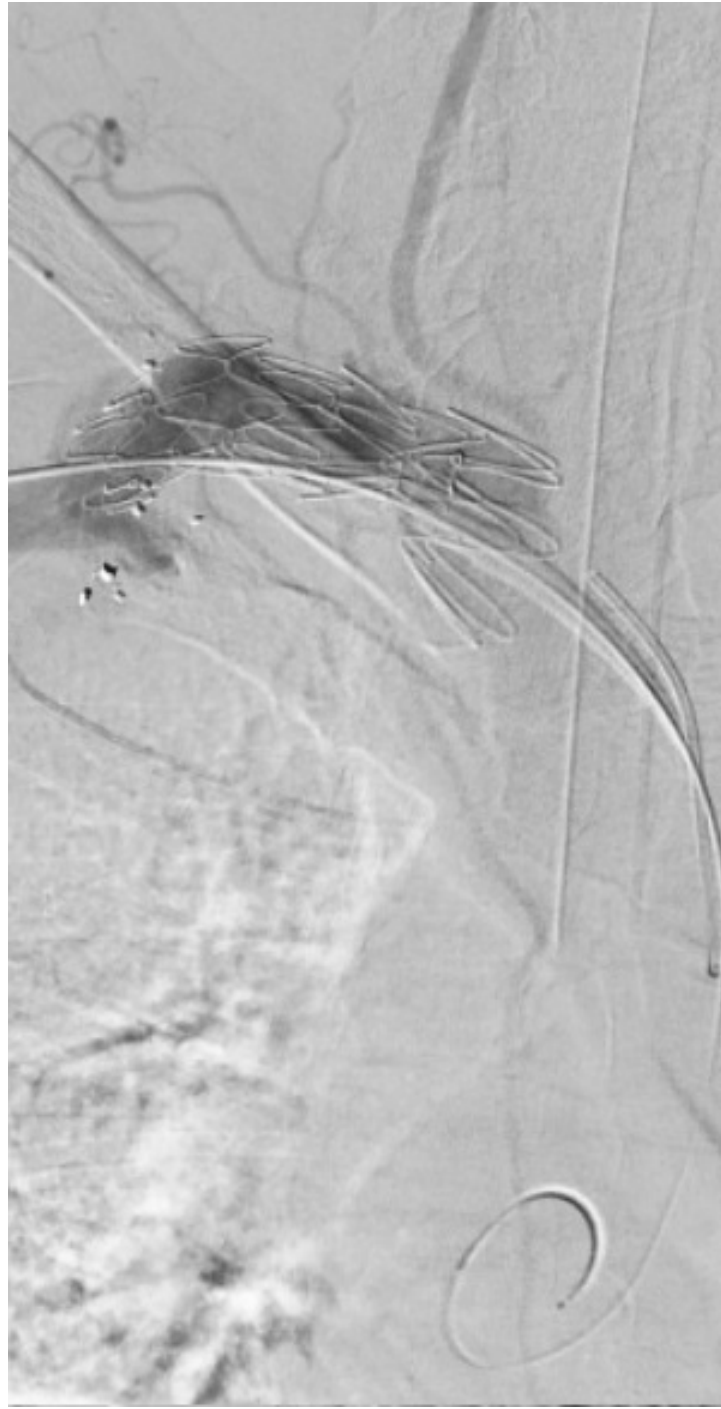

Figura 4. Angiografia por subtração digital evidenciando posicionamento de endoprótese monoilíaca $26 \times 14 \times 90 \mathrm{~mm}$ Braile (Braile Biomédica, São José do Rio Preto, Brasil) em artéria subclávia aneurismática, posterior à saída da artéria vertebral direita que foi mantida pérvia pelo free flow.

Equalizer, com selamento imediato da FAV, tratamento de dilatação aneurismática de artéria subclávia e manutenção da perviedade da AVD pelo free flow da endoprótese.

No terceiro dia após a abordagem endovascular, o paciente foi submetido ao desbridamento de tecidos desvitalizados e recebeu alta hospitalar após 7 dias com prescrição de ciprofloxacino e clindamicina para completar o ciclo de 14 dias, além de dupla antiagregação com ácido acetilsalicílico (AAS) $100 \mathrm{mg} /$ dia, clopidogrel $75 \mathrm{mg} /$ dia e faixa elástica de média compressão (20-30 mmHg) no MSD. No retorno ambulatorial de 30 dias, observou-se diminuição

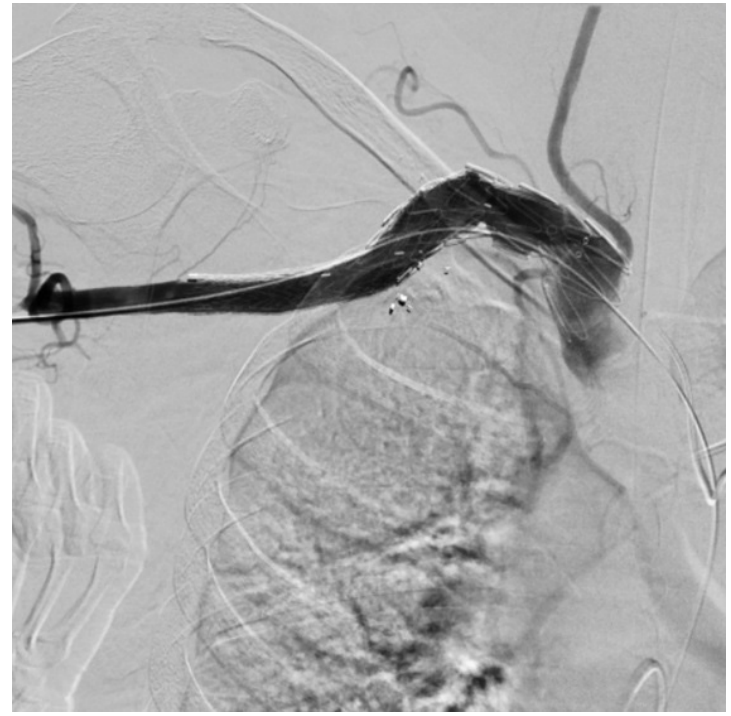

Figura 5. Angiografia por subtração digital evidenciando posicionamento de endoprótese monoilíaca $16 \times 16 \times 95 \mathrm{~mm}$ Excluder $^{\circledast}$ (WL Gore \& Associates, Flagstaff, EUA) com sobreposição na primeira prótese. Nota-se a manutenção da artéria vertebral direita.

do edema e dos sinais de hipertensão venosa, além de cicatrização das lesões tróficas, mantendo apenas a delimitação da necrose em falange distal do $3^{\circ}$ quirodáctilo, a qual foi amputada (Figura 6). $\mathrm{Na}$ avaliação com 10 meses de pós-operatório, foi realizada ecografia vascular com Doppler (EVD) arterial e evidenciado as endopróteses pérvias e sem estenoses hemodinamicamente significativas, além de angiotomografia confirmando achados de normalidade do ultrassom (Figura 7). Em última avaliação ambulatorial com 25 meses de pós-operatório, o paciente apresentava-se sem queixas, com feridas cicatrizadas, sem edema residual e com pulsos radial e ulnar palpáveis.

\section{DISCUSSÃO}

O tratamento cirúrgico convencional para lesões no território dos vasos subclávios e axilares é complexo, muitas vezes envolvendo incisão supra e/ou infraclavicular, e, para controle proximal, pode ser necessário esternotomia mediana para acessar a artéria subclávia direita ou toracotomia anterolateral no $3^{\circ}$ ou $4^{\circ}$ espaço intercostal para a esquerda ${ }^{5,6,10}$.

Diante disso, o tratamento endovascular torna-se opção atraente. Para o tratamento das FAVs traumáticas, há a possibilidade de utilização de stents recobertos ou endopróteses periféricas ${ }^{2,5,6,8,11,12}$, com menor tempo cirúrgico e menor perda sanguínea ${ }^{1}$.

Particularmente, neste caso, a utilização da endoprótese aórtica foi a melhor opção na opinião dos 


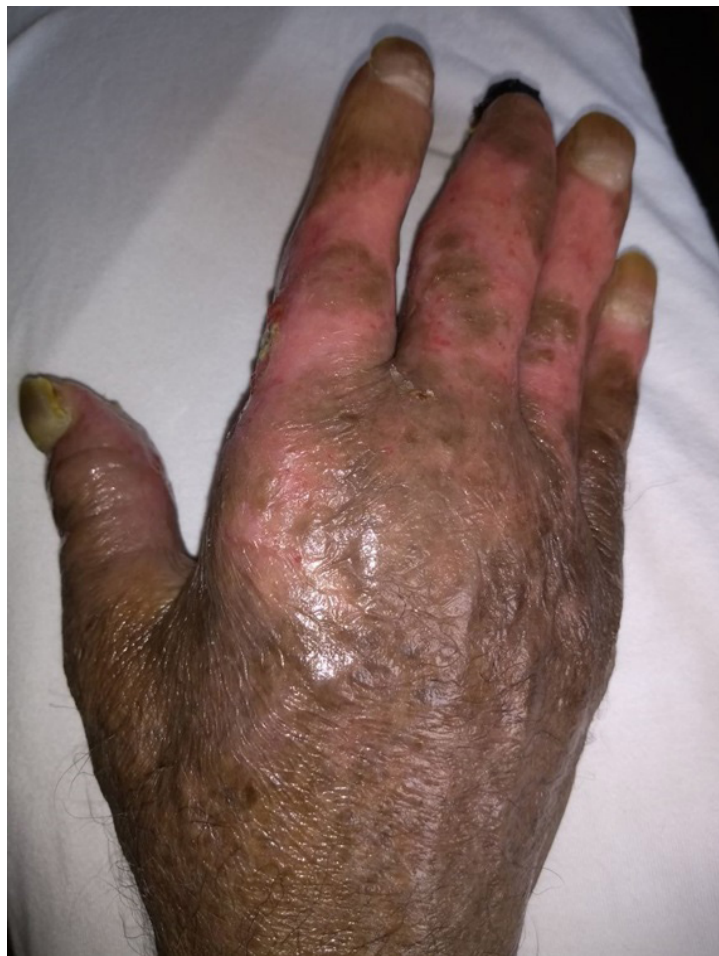

Figura 6. Aspecto da mão direita após 30 dias, evidenciando cicatrização das lesões tróficas, mantendo apenas necrose em falange distal do $3^{\circ}$ quirodáctilo.

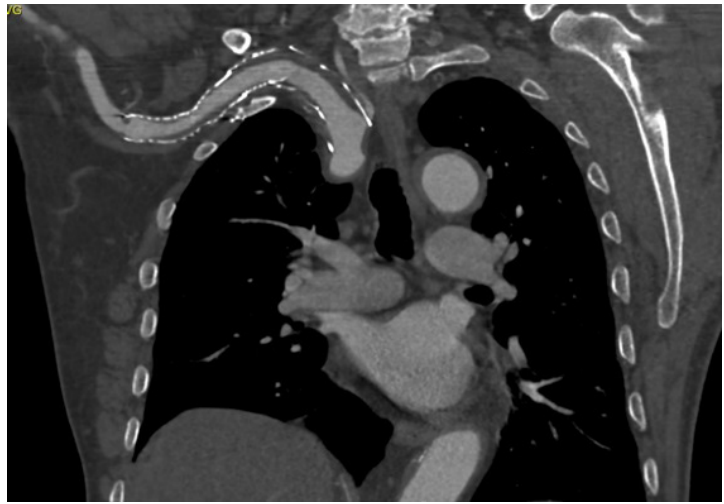

Figura 7. Corte coronal de angiotomografia de controle após 10 meses, evidenciando endopróteses pérvias e sem endoleaks. Nota-se artéria vertebral direita pérvia.

autores, pois a abordagem cirúrgica aberta convencional está relacionada com maior morbimortalidade. Não foi aventada a possibilidade de utilização do stent revestido devido ao diâmetro e comprimento do aneurisma da artéria subclávia, pois os materiais disponíveis até o momento apresentam diâmetros e extensão limitados. A embolização com plugue vascular poderia ser suficiente para o tratamento da FAV, porém, devido ao diâmetro do aneurisma, o paciente poderia evoluir com trombos intraluminais e consequente embolização distal, além do aumento do diâmetro e compressão de estruturas adjacentes ou rotura do aneurisma.

Além do sucesso cirúrgico, cita-se a correção da hipertensão pulmonar e dos sintomas relacionados à FAV, como a insuficiência cardíaca congestiva secundária, mesmo em forma grave ${ }^{9}$. A longo prazo, ainda não se estabeleceu a durabilidade de próteses endovasculares nesse segmento; portanto, não há consenso do uso rotineiro do tratamento endovascular ${ }^{2}$. Em estudo recente avaliando o tratamento endovascular para lesões axilo-subclávias, foi encontrada mortalidade intra-hospitalar de $5,4 \%$ e patência primária do stent de $88 \%$ no período médio de 13,2 meses 5 .

Os traumas vasculares da extremidade superior com formação de FAV são raros, de curso insidioso e de difícil diagnóstico na sua fase inicial. A abordagem endovascular com uso de stents revestidos é segura, podendo ser empregada. No entanto, no caso relatado, o diâmetro e comprimento do aneurisma limitaram seu uso. Portanto, apresentamos a possibilidade de utilização de endoprótese aórtica em situação incomum e de exceção para tratamento de FAV na extremidade superior, com sucesso terapêutico.

\section{REFERÊNCIAS}

1. Mo A. Endovascular repair of traumatic arteriovenous fistula between axillary artery and vein. Chin J Traumatol. 2014;17(2):1124. PMid:24698582.

2. Maués JJB Fo, Hauter HL. Tratamento endovascular de fístula traumática de vasos subclávios: relato de caso. J Vasc Bras. 2018;17(3):248-51. http://dx.doi.org/10.1590/1677-5449.010317. PMid:30643512.

3. Cohen JE, Rajz G, Gomori JM, et al. Urgent endovascular stent-graft placement for traumatic penetrating subclavian artery injuries. J Neurol Sci. 2008;272(1-2):151-7. http://dx.doi.org/10.1016/j. jns.2008.05.016. PMid:18649895

4. Castelli P, Caronno R, Piffaretti G, et al. Endovascular repair of traumatic injuries of the subclavian and axillary arteries. Injury. 2005;36(6):778-82. http://dx.doi.org/10.1016/j.injury.2004.12.046. PMid:15910833.

5. Branco BC, DuBose JJ. Endovascular solutions for the management of penetrating trauma: an update on REBOA and axillo-subclavian injuries. Eur J Trauma Emerg Surg. 2016;42(6):687-94. http://dx.doi. org/10.1007/s00068-016-0739-5. PMid:27853843.

6. Jaldin RG, Bertanha M, Sobreira ML, et al. pseudoaneurisma da artéria subclávia próximo à origem da artéria vertebral após punção inadvertida: Tratamento endovascular ou cirurgia aberta? J Vasc Bras. 2013;12(3):237-42. http://dx.doi.org/10.1590/jvb.2013.039.

7. Oliveira PPM, Petrucci O, Vilarinho KADS, Silveira LM, Vieira RW, Braile DM. Fístula traumática entre tronco braquiocefálico e veia braquiocefálica por arma de fogo. Arq Bras Cardiol. 2008;90(4):213. http://dx.doi.org/10.1590/S0066-782X2008000400013.

8. Santos EPJr, Batista RRA, Felici FM, Correia VE, Oliveira MB, Alves RF. Endovascular correction of a traumatic internal iliac arteriovenous 
fistula with a covered stent. J Vasc Bras. 2014;13(1):48-52. http:// dx.doi.org/10.1590/jvb.2014.010.

9. Pilan BF, Oliveira AM, Siqueira DED, Guillaumon AT. Tratamento de fístula arteriovenosa adquirida com repercussões hemodinâmicas graves: desafio terapêutico. J Vasc Bras. 2014;13(1):34-8. http:// dx.doi.org/10.1590/jvb.2014.007.

10. Medeiros CAF, Landim RM, Castro AN, et al. Condutas no trauma penetrante da artéria axilar. J Vasc Bras. 2003;2(3):225-8.

11. Ramacciotti E, Gerardi VA Fo, Fagundes DJ. Endovascular de Fístulas Artériovenosas. Acta Cir Bras. 1999;14(1):47-51. http:// dx.doi.org/10.1590/S0102-86501999000100009.

12. Medeiros CAF, Hatsumura TC, Gusmão DR, Freire LMD, Rocha EF, Guillaumon AT. Tratamento endovascular do trauma arterial dos membros. J Vasc Bras. 2008;7(1):56-61. http://dx.doi.org/10.1590/ S1677-54492008000100010.
Correspondência Rodrigo Gibin Jaldin

Universidade Estadual "Júlio de Mesquita Filho" - UNESP, Departamento de Cirurgia e Ortopedia Av. Prof. Mário Rubens Guimarães Montenegro, s/n, Campus de Botucatu

CEP 18618-687 - Botucatu (SP), Brasil Tel.: (14) 3880-1446 E-mail: rodrigo.gibin@unesp.br

Informações sobre os autores

VTRSG - Residente de Cirurgia Vascular, Hospital das Clínicas, Faculdade de Medicina de Botucatu, Universidade Estadual "Júlio de Mesquita Filho" (UNESP).

RG) - Professor, Disciplina de Cirurgia Vascular, Faculdade de Medicina de Botucatu, Universidade Estadual "Júlio de Mesquita Filho" (UNESP).

FDR - Ex-residente de Cirurgia Vascular, Hospital das Clínicas, Faculdade de Medicina de Botucatu, Universidade Estadual "Júlio de Mesquita Filho" (UNESP).

MTSS - Médica Assistente, Disciplina de Cirurgia Vascular, Faculdade de Medicina de Botucatu, Universidade Estadual "Júlio de Mesquita Filho" (UNESP). REFP - Médico Assistente, Disciplina de Cirurgia Vascular, Faculdade de Medicina de Botucatu, Universidade Estadual "Júlio de Mesquita Filho" (UNESP).

MB - Professor Assistente Doutor, Disciplina de Cirurgia Vascular, Faculdade de Medicina de Botucatu, Universidade Estadual "Júlio de Mesquita Filho" (UNESP).

MLS - Professor Livre-Docente, Disciplina de Cirurgia Vascular, Faculdade de Medicina de Botucatu, Universidade Estadual "Júlio de Mesquita Filho" (UNESP).

WBY - Professor Titular, Disciplina de Cirurgia Vascular, Faculdade de Medicina de Botucatu, Universidade Estadual "Júlio de Mesquita Filho" (UNESP).

Contribuições dos autores Concepção e desenho do estudo: VTRSG, RGJ, MB, WBY Análise e interpretação dos dados: VTRSG, RGJ, MTSS, REFP Coleta de dados: VTRSG, RG], MTSS, FDR Redação do artigo: VTRSG, RG), MLS Revisão crítica do texto: VTRSG, RG), FDR, MLS, WBY Aprovação final do artigo*: VTRSG, RGJ, FDR, MTSS, REFP, MB, MLS,

WBY

Análise estatística: N/A.

Responsabilidade geral pelo estudo: VTRSG, RGJ, FDR, MLS

*Todos os autores leram e aprovaram a versão final submetida ao J Vasc Bras. 